\title{
2. GOBERNABILIDAD Y DEMOCRACIA
}

\section{DE LA GOBERNABILIDAD*}

\author{
Manuel Alcántara Sáez**
}

1.

Los estudios sobre la acción de gobierno o, en términos más clásicos, del Poder Ejecutivo, la forma en que ésta se adopta y se pone en marcha, sus características, sus consecuencias y la percepción de las mismas por parte de los destinatarios finales requieren de una buena dosis de pluridisciplinariedad en el seno de las ciencias sociales. También se hace necesaria la utilización del enfoque comparativo, tanto en su propia acepción en cuanto método de estudio como por la aceptación de la idea de que los problemas no son exclusivos de una sola sociedad. Sin ambos elementos cualquier aproximación corre el riesgo de no alcanzar óptimos resultados. Esto es aun más evidente cuando se constata que en la realidad internacional de la última década los cambios políticos hacia la democratización van de la mano de transformaciones económicas que suponen procesos de liberalización definidos por profundas mutaciones en el papel del Estado, por el predominio de las reglas de juego que impone el mercado, por la desregularización y la apertura al comercio y al capital financiero internacional y por los efectos del nivel alcanzado por el cambio tecnológico.

En las páginas que siguen va a prestarse especial atención a la conceptualización de lo que se entiende por gobernabilidad en el ámbito de un sistema político. Término que adquiere una connotación imprecisa y ambigua, de "palabra atrapatodo" -"catch-all word" (Flisfisch, 1989: 113)-, o de "concepto elusivo" (Tomassini, 1993:11), y que refiriéndose a un tema de estudio clásico ha cobrado interés en los últimos años, de manera que ha llegado a introducirse en las agendas de los principales foros de discusión planetarios. En efecto, el concepto de gobernabilidad se ha desarrollado a lo largo de las tres últimas décadas en torno a varios esca-

(*) La versión aquí recogida forma parte del libro de próxima aparición Gobernabilidad crisis y cambio, editado por el Fondo de Cultura Económica de México y el Centro de Estudios Constitucionales de Madrid.

(**) Catedrático de Ciencia Política, Facultad de Ciencias Sociales de la Universidad de Salamanca. lones profundamente diferentes. El primero de ellos lo representaron los distintos escritos sobre la "crisis de la democracia"; posteriormente entró en liza la corriente intelectual preocupada con el rendimiento del sistema político, tanto en la esfera económica como en la institucional; también otro escalón fue el conformado por el debate en torno a la crisis del Estado; por último, en cuanto a su capacidad de alumbrar un modelo de desarrollo que facilitase la prosperidad de los países menos avanzados. En un momento inicial se lleva a cabo una revisión de parte de la bibliografía sobre el tema para adoptar una definición concreta válida con respecto al conjunto del texto.

El objetivo primordial, por consiguiente, consiste en introducir los temas de Ciencia Política que se refieren a las condiciones que afectan a la acción del gobierno. Estas condiciones se encuentran en el seno del propio Gobierno en cuanto que son parte de su propio funcionamiento $\mathrm{y}$, asimismo, aparecen en el entorno del mismo. Las primeras se refieren al modo en que se conforma el gobierno desde la perspectiva del juego de partidos y del sistema electoral, y al proceso de toma de decisiones, donde concurren las relaciones entre los poderes del Estado, el estilo de las políticas públicas puestas en marcha y el acceso de diferentes grupos de corte socioeconómico. Las segundas comportan un conjunto de elementos integrados por el legado histórico de la sociedad, la composición de las rupturas o dimensiones (clivajes) de la misma, las características de su estructura económica y el tipo de engarce que mantiene con el escenario internacional.

Se trata, por tanto, de una tarea que difícilmente puede aportar teorías explicativas unicausales, sino más bien un entramado de causalidades movibles, algunas veces cuestionables y difíciles de comprobar empíricamente como explicaciones de valor universal. La complejidad que entrañan los factores explicativos de la gobernabilidad, o el hecho de ser precisamente "el producto único de fuerzas diversas" (Arbós y Giner, 1993: 66), se ve conformada tanto por aquellos productos que provienen del Gobierno en forma de políticas públicas, como por las respuestas que 
éste recibe de la sociedad, bien de estratos de individuos organizados o bien actuando individualizamente, de manera que una aproximación sistémica al fenómeno parece apropiada. El flujo constante existente entre uno y otro polo queda condicionado por un número muy elevado de aspectos. Sin embargo, de acuerdo con las principales líneas de análisis desarrollado por las ciencias sociales, el conjunto de factores explicativos del mayor o menor grado de gobernabilidad se circunscribe a una cifra relativamente baja de condiciones que se indican a lo largo de las páginas siguientes cuyo carácter es el de variables independientes.

2.

La base inicial de la que se parte es la aceptación de la legitimidad democrática como la única plausible. Dicho tipo de legitimidad es entendida tanto como una situación "de hecho" basada en la ausencia de desafíos concretos, como en la aceptación consciente y mayoritaria por parte de la ciudadanía de unas determinadas reglas. Como señala Peters (1987: 256), de alguna manera el fenómeno de la gobernabilidad es inherente a la democracia representativa por el desfase que ésta conlleva en el momento de trasladas el mandato electoral recibido de los votantes en políticas que pudieran ponerse en marcha de una manera concreta y con unos resultados determinados. Idea parecida a la que señala que, bajo condiciones democráticas, la gobernabilidad es problemática debido "a la posibilidad efectiva de la alternancia de partidos distintos en el Gobierno y del hecho de que la reproducción del orden democrático en el tiempo exige la preservación de esa posibilidad" (Flisfisch, 1989: 115). Pero, a su vez, la gobernabilidad en sistemas democráticos representa una situación especial por cuanto que la democracia respeta la lógica de la igualdad política mientras que la gobernabilidad respeta la del poder (Coppedge, 1993).

De esta manera, aunque la gobernabilidad es un fenómeno relativo a cualquier tipo de régimen, en el presente trabajo se centra fundamentalmente en los de ámbito democrático. El amplio alcance de dicho ámbito, dentro de una perspectiva histórica, es algo muy reciente. La extensión de los sistemas democráticos a un elevado número de países, sin llegar todavía a la mayoría, que comienzan una insólita andadura bajo imperativos formales algo o totalmente desconocidos hasta la fecha por sus sociedades, permite referirse, por primera vez en la historia, a una forma casi similar de gobierno. Por consiguiente, se presentan unas condiciones semejantes para la acción de gobierno, que atañen a una cincuentena de pases (Huntington, 1991). No obstante, también puede plantearse el tema de la gobernabilidad en sistemas que no son democráticos. La justificación de dicha opción se basara en el interés posterior en desarrollar un análisis de la gobernabilidad en sistemas políticos de países que abandonaron su carácter no democrático recientemente. Todo ello, porque se estima que ciertos aspectos que configuran una crisis de gobernabilidad ya se encontraban presentes al iniciarse las transiciones políticas.

Por otra parte, existen dos tipos de elementos que se re- lacionan con la gobernabilidad por ser los de la propia consolidación de todo sistema político, y cuya característica fundamental es la de su interrelación. El primero de ellos, sin pretender establecer ningún orden de prelación, es la legitimidad del sistema político, entendida en su vertiente estricta de creencia en que las instituciones existentes son mejores que otras que pudieran haber sido establecidas, y que, por tanto, el Gobierno puede exigir obediencia. En segundo término, la eficacia, en el doble aspecto que requiere la capacidad de un régimen para encontrar soluciones a problemas: en cuanto a la idoneidad de las políticas diseñadas por el Gobierno para reducir al mínimo el conflicto, asegurar una tendencia a la repartición de la riqueza y propiciar su incremento; y con respecto a la capacidad operativa del Gobierno de actuar sin sobresaltos, pudiendo realizar sus programas y no estando sujeto a elementos disfuncionales.

Ahora bien, un aspecto final dentro de estas consideraciones preliminares lleva a plantear cierta relativización de la política en cuanto al papel que pudiera desempeñar el "buen gobierno" en una sociedad. El variopinto entramado conformador de un régimen político no es un medio que por sí mismo necesariamente tienda a contribuir a la construcción de una sociedad más justa, igualitaria e integrada por individuos libres y más felices, pero sí lo puede ser para evitar que ésta se deshaga. Es desde esta percepción desde donde debe valorarse el significado que representa que la gobernabilidad de un sistema se sitúa en un continuo en el que lo verdaderamente preocupante es traspasar los límites, a veces solo reversibles a un costo muy oneroso, en los que se entra en un estadio de ingobernabilidad con los consiguientes resultados negativos. El síndrome yugoslavo o de varias de las nuevas repúblicas independientes que conformaban la hoy desaparecida Unión Soviética es, en este sentido, desafortunadamente revelador.

\section{3.}

El interés sobre el término de gobernabilidad se encuentra ligado a la aparición o agudización de situaciones de crisis en los sistemas políticos. Estas crisis de gobernabilidad se prestan a múltiples interpretaciones, entre las que destacarían dos como las más relevantes y cuyo carácter es contrapuesto. Se trata de la comprensión de la crisis de gobernabilidad como incapacidad de los gobernantes insertos en las "condiciones contradictorias de los sistemas capitalistas" y, en segundo término, como consecuencia de las demandas excesivas de los ciudadanos (Pasquino, 1988: 192). Además, en este último caso, el número y la intensidad de las fracturas y dimensiones (clivajes) pueden llegar a generar una situación aún más compleja. Si bien tienen algunos puntos en contacto, tienden a degenerar en acusaciones, convirtiéndose en punta de lanza de perspectivas ideológicas enfrentadas, circunstancia que debe tenerse en cuenta a la hora de llevar a cabo cualquier análisis.

Ambas concepciones ponen de relieve lo complejo de las relaciones que se establecen al interior de un sistema político y del juego de factores que influyen en su gober- 
nabilidad, pero comparten el criterio general que la entiende como el grado en el que el relativo poder de los grupos más relevantes en la arena pública se encuentra respetado por las instituciones ya sean formales o informales del proceso político (Coppedge, 1993: 2). En este equilibrio descansa, pues, el éxito de la acción de gobierno. De este modo, resulta fundamental el estudio de la manera en la que el Gobierno se ocupa y desarrolla sus funciones así como su relación con la sociedad. Circunstancias que avalan la utilización teórica del concepto de sistema político como entramado en el que se dan cita elementos institucionales, valores conformados por la evolución histórica de la comunidad, actores sociales y el medio internacional.

Desde una perspectiva preocupada por el establecimiento de indicadores que marquen el nivel de gobernabilidad de un sistema político, aquí se han tomado cinco conceptualizaciones de entre la diferente literatura sobre el tema que se conecta con las concepciones citadas. La primera señala la existencia de cuatro notas generales que caracterizan una situación de ingobernabilidad y que son: 1) la indisciplina, manifestada "en los esfuerzos que realizan determinados ciudadanos para influir en las decisiones públicas por métodos violentos, ilegales o anómalos"; 2) la inestabilidad, en cuanto fracaso de los intentos de la élite política "para conservar sus posiciones de dominación o para reproducir las coaliciones preexistentes"; 3) la ineficacia, entendida como la disminución de la capacidad de políticos y burócratas "para alcanzar los objetivos deseados y asegurar el acatamiento de ellos por medio de medidas de coordinación obligatorias o de decisiones emanadas de la autoridad del Estado; y 4) la ilegalidad, es decir, los esfuerzos realizados por los poseedores de un poder corporativo de alto nivel "para evadir restricciones legales y constitucionales en búsqueda de ventajas e incluso de su propia supervivencia" (Schmitter, 1988:375).

Por otro lado, Sundquist(1) establece, en una segunda conceptualización, cuatro características que afectan la capacidad de gobierno. La primera es el grado de calidad de la burocracia que garantice de forma suficiente asesoramiento y liderazgo en el proceso de formulación y puesta en marcha de las políticas. La segunda se refiere al compromiso de la burocracia con los objetivos del Gobierno del momento en contraposición con sus intereses en tanto que organización y que individuos. En tercer lugar, el entorno institucional en el proceso de gestación de políticas por parte del gobierno puede influir en la gobernabilidad. Por último, se señala que el sistema de partidos puede asimismo influir en la gobernabilidad, especialmente en lo relativo a la habilidad para generar y poner en marcha mandatos claros de acción.

Coppedge (1993: 13) enuncia seis condiciones de la gobernabilidad, centrando las dos primeras en su ámbito general (la capacidad y el deseo de todos los grupos políticamente relevantes para comprometerse con algún tipo de arreglo institucional que sirviese para dirimir sus diferencias) y las restantes en la gobernabilidad democrática. Estas

(1) Citado en Peters (1987) cuatro últimas se refieren a: 1) la aceptación de compromisos institucionales democráticos que confieren gran peso a los grupos de masas políticamente relevantes (básicamente los partidos políticos) por parte de aquellos otros de carácter más elitista (Iglesia, empresarios, militares,...); 2) la aceptación de arreglos que permitan la representación efectiva de estos últimos grupos por parte de la clase política elegida; 3) la efectiva representación de los ciudadanos por esta clase política elegida; y 4) la creación y el mantenimiento de mayorías que funcionen basadas en criterios partidistas como consecuencia de decisiones tomadas por la clase política.

Tomassini (1993: 12) hace depender la gobernabilidad de tres variables: el fortalecimiento de la sociedad civil y la evolución de la cultura política, la orientación y el comportamiento de la economía, y la integración de sectores cada vez más amplios de la sociedad en el sistema productivo. Este autor llega a señalar un extenso número de "condiciones para fortalecer la gobernabilidad" (33) que sitúa en los tres escenarios que representan: los organismos de gobierno encargados de manejar la economía y al sector publico; el conjunto de las organizaciones del Estado, incluyendo la interacción entre los organismos económicos y políticos del Poder Ejecutivo, sus relaciones con los demás poderes públicos, y con otras instituciones y procesos a los cuales el Estado está vinculado; y el tercero incluye la interacción entre una amplia gama de actores pertenecientes a la sociedad civil organizada, a la economía y al mercado, y a los sectores sociales menos favorecidos (Tomassini, 1993: 71).

Finalmente, la constatación de que la gobernabilidad es un fenómeno pluridimensional lleva a plantear por parte de Arbós y Giner (1993: 14) la existencia de cuatro niveles, como mínimo, en los que se mueven los procesos complejos de la gobernabilidad de las sociedades. Se trata del dilema legitimidad-eficacia; de las presiones y demandas del entorno gubernamental, o de la carga de responsabilidades; de la reestructuración corporativa de la sociedad civil; y de la expansión y cambio tecnológico, con sus repercusiones demográficas, ecológicas y sociales consiguientes.

4.

A la hora de avanzar en la comprensión del fenómeno de la gobernabilidad en el marco de teorías explicativas Birch (1984) y Pasquino (1988) coinciden en indicar la existencia de tres hipótesis o corrientes intelectuales existentes en el seno de los Estados occidentales industrializados, que contribuyen a explicar el tema de la gobernabilidad(2). Sus categorizaciones, en todo caso, son bastante complementarias y aparecen en buena medida integradas en la posición de Bourricaud (1992) que se aborda en cuarto lugar.

La primera corriente referida a la gobernabilidad, deri-

(2) Arbós y Giner (1993: 9-11) concuerdan también casi plenamente al dibujar cuatro concepciones tradicionales de gobernabilidad: la de los conservadores, la de los neoconservadores, la de los liberales y la de los marxistas. 
vada de un planteamiento neoconservador liderado, entre otros, por Daniel Bell y Samuel Huntington, explica su posición negativa (la ingobernabilidad) como el producto de una sobrecarga de demandas a las que el Estado responde con la expansión de sus servicios y de su intervención provocando inevitablemente una crisis fiscal. La acusación que se vierte sobre las políticas del Estado del bienestar es haber enfatizado los derechos y dado mayor importancia a la igualdad, además de subrayar el debilitamiento de la familia y de los vínculos comunales dejando que la gente dependiera de las agencias gubernamentales. De manera más concreta, el diagnóstico de la situación lleva a plantear la existencia de cuatro tendencias que generan disfunciones en el sistema democrático (Crozier, Huntington y Watanuki, 1975: 161,171) y que se refieren: a la deslegitimación de la autoridad y a la pérdida de confianza en el liderazgo como consecuencia de la persecución de las virtudes democráticas de la igualdad y del individualismo; a la "sobrecarga" del gobierno debido a la expansión de la participación política y del desarrollo de las actividades gubernamentales; a la desagregación de intereses como consecuencia de la intensificación de la competencia política y que ha conducido a la fragmentación de los partidos políticos; y al incremento del parroquialismo nacionalista en la política exterior dadas las presiones ejercidas por la sociedad. Todas estas tendencias, según señala tiempo después Hirschman (1991: 148) son la imagen prototípica de la "tesis del riesgo". Es decir, "la insistencia en el riesgo de dañar un logro anterior con alguna acción", ya que estos tres autores lo que vienen a mantener es que la vitalidad de la democracia en los pases industrializados en la década de los años sesenta produce un aumento considerable de la actividad gubernamental y una consiguiente disminución notable de la autoridad gubernamental.

La segunda categoría es la formulada por economistas neoliberales, políticamente neoconservadores, cuyo principal punto de partida se basa en el presupuesto de la mala relación existente entre el keynesianismo y la democracia, de suerte que "la yuxtaposición de las prescripciones de la política keynesiana y la democracia política crea una mezcla inestable" (Buchanan y Wagner, 1983: 126). En ese sentido, señalan que la democracia se encuentra presionada y que llega a generar una rápida inflación cuando los gobiernos se ven confrontados por los sindicatos y por los votantes que consideran al Estado como un botón de servicios inagotables. La consecuencia de un análisis según el cual los políticos son percibidos como unos dilapidadores empedernidos, "empeñados en una competencia continua, gastan más que lo que recaudan" (Buchanan y Wagner, 1983: 160), es que se debe actuar sobre la inflación bien con medidas monetarias o con otras de tipo presupuestario. Con respecto a las primeras, se precisa que la inflación es consecuencia del déficit, por cuanto las instituciones monetarias hacen que la oferta monetaria sea variable en relación al déficit. Ahora bien, no se produciría una situación inflacionaria si hubiese un aumento de la oferta monetaria con relación a la oferta de bienes (Buchanan y Wagner, 1983: 101,102). En cuanto a la actuación en el escenario presupuestario, se lleva a cabo mediante el establecimiento de impuestos crecientes y de controles sobre precios y salarios.
En cualquiera de los dos casos se constató que se trataba de una visión excesivamente coyuntural puesto que las tasas de inflación se redujeron y permanecieron estables en la década siguiente a 1983. Por otra parte, tampoco se llegó a experimentar el citado control, ni se incrementó la presión impositiva.

En tercer lugar, la corriente neomarxista queda de manifiesto en el trabajo de O'Connor, por un lado, y de Habermas y Offe por otro. O'Connor (1973) se refiere a la "crisis fiscal del Estado" resultante de las contradicciones del Estado del "capitalismo avanzado" agravadas por el sistema democrático. Por su parte, Habermas (1978: 70,71) mantiene la existencia de una doble crisis: "de entrada", en su acepción de crisis de legitimidad clásica, y "de salida" como crisis de racionalidad, en el momento en el que la burocracia no hace compatibles, o es incapaz de manejar correctamente, los mecanismos de control que le exige el sistema democrático. A ojos de los trabajadores el sistema capitalista está legitimado por el Estado liberal democrático que suplanta el papel dominante de la clase capitalista. En este tipo de Estado los valores políticos de esta clase gozan de lo que Gramsci llamó "hegemonía ideológica", y por tanto ambos establecen los términos principales del debate político e indican los canales y los límites en los que se confina normalmente al debate político. El Estado liberaldemocrtico adopta varias políticas y tácticas para proteger tanto su propia legitimidad como la del orden social desarrollándose para ello servicios asistenciales. La situación final lleva a que este sistema de legitimación empiece a quebrarse apareciendo crisis fiscales, conflictos entre diferentes tipos de trabajadores según su rama de producción, conflictos regionales y conflictos étnicos (Birch, 1984). De todas estas amenazas, en los países industrializados solamente ha adquirido una tibia presencia el conflicto en torno al replanteamiento de un nuevo modelo federal en Canadá.

Por último, Bourricaud (1992) señala que si se parte de la definición del concepto de gobernabilidad como un conjunto de condiciones que aseguren el ejercicio del poder en una sociedad sin excesivo riesgo de que ésta llegue a desarticularse violentamente, debe aceptarse la existencia de ciertas condiciones que preserven un mínimo de coherencia y de unidad en las sociedades. De esta forma, la discusión se centra en un terreno clásico en el que predomina el sentimiento de pertenencia al mismo cuerpo político y la creencia de que hay una ley implícita común que todos los ciudadanos están obligados a respetar. Las condiciones no serían otras que la existencia de un sistema normativo respetado por las autoridades centrales con el uso ocasional de sanciones efectivas y, en segundo término, la congruencia entre el sistema normativo y las motivaciones de los actores. Ahora bien, dichas condiciones deberían verse complementadas por dos principios: la consecución del grado más alto de productividad y la mejor, cuanto no la más justa, distribución. Sin embargo, con relación a la sociedad de los países desarrollados al filo de 1990, Bourricaud denuncia la posibilidad de que las crisis de gobernabilidad sean consecuencia directa de la gestación y evolución de minorías vehementes ("impassioned minorities") que rompan el equilibrio del cuerpo político como consecuencia de distin- 
tos aspectos que podrían enmarcarse desde la crisis de valores, a la difícil relación entre productividad y solidaridad, pasando por el peligro de la anomia social. Dichas minoras, surgidas en un espacio común al que Bourricaud denomina Babel y que no es sólo un espacio de confusión sino también de confrontación, son actores colectivos intermedios entre los grupos de interés y los movimientos sociales. Su presión violenta puede eventualmente terminar, en el más extremo de los casos, en terrorismo o en la exacerbación del comportamiento del "gorrón" ("free rider") al tratar de obtener los beneficios de la cooperación sin asumir ningún tipo de los costes (Bourricaud, 1992: 47).

\section{5.}

En el caso de los sistemas políticos democráticos de los países en vías de desarrollo, se da una situación teórica en que si bien "la problemática de la gobernabilidad tiene una historia muy breve" (Mayorga, 1992: 17), existen razones sobradas para estimar que la acción de gobierno en sí misma considerada es algo trascendental que sobrepasa la actual coyuntura democratizadora, de manera que algunos problemas de la gobernación son anteriores (Coppedge, 1993: 2). En dicho medio, tanto la aproximación estructural-funcionalista como la neo-marxista han tendido a converger en un nivel similar de causación a la hora de explicar las crisis de gobernabilidad (Kohli, 1990: 24). Desarrollistas y dependentistas han contemplado la emergencia del desorden (o del conflicto de clases) como un resultado de la creciente dislocación entre los aspectos subjetivos de comprensión de la estructura social y objetivos de la posición en que dicha estructura tiende a crear una sobredemanda o un conflicto de clases en el sistema político; eso es, del fracaso de los valores prevalecientes (ideologías) para legitimar la división del trabajo existente (la estructura de clase). Para los estructural-funcionalistas las fuerzas motrices de dichos cambios han sido el desarrollo económico y su concomitante modernización, mientras que para los marxistas se trataba del capitalismo. En ambas visiones se producía una obvia inclinación en favor de centrar en la sociedad las explicaciones de la crisis de gobernabilidad.

Sin embargo, en las democracias del Tercer Mundo se dan condiciones políticas especiales que realzan el significado de las variables políticas para entender las recurrentes crisis de gobernabilidad. De esta suerte, pueden asumirse como tales: la calidad del liderazgo y las preferencias de éste, la ideología prevaleciente, el grado de armonía interélites, el diseño de las instituciones políticas dominantes como el sistema de partidos y las relaciones entre el Ejecutivo y el Legislativo, y el peculiar papel del Estado como responsable primordial del desarrollo socioeconómico (Kohli, 1990: 29). A todas ellas cabría añadir el grado de corrupción, no únicamente patrimonio de los países en vías de desarrollo pero sí con una mayor capacidad de impacto, que el sistema puede llegar a aceptar como consecuencia de su gravitación en el descontento social. No obstante, es precisamente la capacidad interventora del Estado la que por último acentúa las crisis de gobernabilidad bien por su mayor tendencia a politizar la sociedad que la de un Estado limitado, bien por la elevada influencia que se ejerce sobre la economía y sus limitados recursos que hace que se convierta en objeto de una considerable atención política.

Con respecto a América Latina, Coppedge señala la existencia de una evasión recurrente de los mecanismos institucionales a la hora de dilucidar los conflictos entre los grupos políticamente relevantes a los que no son ajenas fuerzas externas que continuamente les influyen haciendo obsoletos tales mecanismos. Esto también es debido al desajuste entre las instituciones formales que no respetan el relativo poder de dichos grupos. Pero, en segundo término, asimismo se registra cierta reluctancia a que estos grupos relevantes se comprometan con algún arreglo institucional. Todo lo cual se sintetiza en que las tradicionales manifestaciones de ingobernabilidad en América Latina no son otra cosa que un desequilibrio en las relaciones de poder entre grupos políticamente relevantes y fórmulas de toma de decisión (Coppedge, 1993:9). Desde otra perspectiva, se ha abogado por una mayor conexión con el ámbito socioeconómico, de manera que se ha indicado que "el problema de la gobernabilidad, que en sus reencarnaciones sucesivas estuvo confinado al ámbito de la política, hoy da aparece cada vez más bajo la forma del contexto o de las condiciones bajo las cuales es posible el desarrollo" (Tomassini, 1993: 11).

6.

La revisión de parte de la literatura existente sobre el concepto de gobernabilidad realizada hasta aquí suministra un cúmulo de elementos suficientemente rico donde enmarcar la definición del concepto de gobernabilidad adoptada en estas páginas. De esta manera, puede entenderse por gobernabilidad la situación en que concurren un conjunto de condiciones favorables para la acción de gobierno que se sitúan en su entorno (de carácter medioambiental) o que son intrínsecas a éste. Por el contrario, ingobernabilidad se refiere a una situación disfuncional que dificulta la actividad y la capacidad gubernamental. Esta definición coincide con la de Leca (1985: 18) al equiparar el concepto de gobernabilidad con la acción de gobierno a la que se le da un carácter positivo, cuando no moralmente satisfactorio, al tratarse de un comportamiento conforme a las reglas "del buen gobierno". Asimismo, la referida conceptualización comparte la visión de Nohlen (1992:4) cuando indica que "el concepto de gobernabilidad se refiere a la interacción entre gobernantes y gobernados, entre capacidades de gobierno y demandas políticas de gobierno. Hace referencia a la tensión que existe entre las dos partes y pone en cuestión el sistema de gobierno, como productor de decisiones políticas y encargado de su ejecución, y su capacidad para estar a la altura de los problemas a resolver". Tanto la adjetivación de "favorables", en la definición establecida, como la del "buen gobierno" de Leca, y el imperativo de Nohlen de poseer una determinada "capacidad para estar a la altura...", reenvían directamente el problema conceptual al marco teórico de la eficacia. Si, complementariamente, el gobierno del que se está hablando, requiere de un tipo definido de apoyo o de aceptación social, el marco que se introduce es 
el de la legitimidad. De esta manera, se dan paso a las definiciones de Flisfisch (1989), Tomassini (1993) y Arbós y Giner (1993). El primero de ellos define gobernabilidad como "la calidad del desempeño gubernamental a través del tiempo" a la que se la deben considerar las siguientes dimensiones: capacidad de adoptar oportunamente decisiones ante eventos que son desafíos que exigen una respuesta gubernamental; efectividad y eficiencia de las decisiones adoptadas; aceptación social de esas decisiones; y coherencia de las decisiones a través del tiempo, de manera que no produzcan efectos contradictorios (Flisfisch, 1989: 113). El segundo autor señala que la gobernabilidad "no sólo se refiere al ejercicio del gobierno, sino además a todas las condiciones necesarias para que esta función pueda desempeñarse con eficacia, legitimidad y respaldo social" (Tomassini, 1993: 6). Los últimos autores, igualmente definen la gobernabilidad como "la cualidad propia de una comunidad política según la cual sus instituciones de gobierno actúan eficazmente dentro de su espacio de un modo considerado legítimo por la ciudadana, permitiendo así el libre ejercicio de la voluntad política del poder ejecutivo mediante la obediencia cívica del pueblo" (Arbós y Giner, 1993: 13).

Por todo ello, el objetivo de análisis queda centrado en el ámbito de lo que hace o deja de hacer el Gobierno, de manera que en el idioma español haya que desarrollar un vocablo redundante como es el de gobernabilidad que sustituye al de la "acción de gobernar" o "gobernación" (governance en inglés) al que se le añade una obligada expectativa positiva que culmina en el "buen gobierno". De ahí que sean las dos esferas indicadas anteriormente relativas al funcionamiento del aparato formal del sistema político y al rendimiento de la política económica, siempre con respecto a sus resultados y a la evaluación social de los mismos, los indicadores de dicho estudio. Ambos conceptos, gobernabilidad e ingobernabilidad, conforman los dos polos de un continuo, de manera que el paso de una situación a otra es gradual y nunca totalmente irreversible.

Complementariamente a la existencia de las condiciones favorables a la acción de gobierno, el propio fenómeno de la gobernabilidad se encuentra sometido a la percepción que de dichas condiciones, y de ciertas disfunciones, entre las que la corrupción política es una de las más frecuentes, tiene la sociedad. De esta suerte, debe incluirse dentro de su análisis la valoración subjetiva llevada a cabo de manera periódica por la misma. Esta respuesta puede formularse tanto por canales institucionalizados como por medios informales. En todo caso, para que su repercusión sobre el propio fenómeno de ingobernabilidad sea significativa, es necesario que alcance una "masa crítica" en función de que las reclamaciones formuladas afecten a elementos "sensibles" del sistema político o conjuguen una movilización social relevante.

Aunque la unidad de análisis es el sistema político, ambos conceptos apenas tendrían que ver, en principio, con la forma política existente que definiera las reglas de funcionamiento del régimen político y las relaciones de sus instituciones más relevantes con la sociedad civil. Unicamente concernerían a la esfera de lo gubernamental entendida como el medio donde se formulan y se ponen en marcha decisiones políticas que afectan al conjunto de la sociedad. Sin embargo, tanto el legado de la bibliografía sobre el tema como la situación mundial en 1993 cuando tienden a generalizarse el sistema político democrático y las formas más abiertas de entendimiento económico, no parecen considerar otra perspectiva acerca del citado binomio antagónico que su ubicación, como ya se expresó anteriormente, en el ámbito del Estado democrático y en una economía de mercado(3) . Todo ello aún teniendo en cuenta las constricciones que registran muchos de los países latinoamericanos y del este europeo a la hora de consolidar sus sistemas democráticos y abandonar el terreno inseguro en el que se encuentran asentados.

En consecuencia, una hipótesis fundamental será que la gobernabilidad quedará aquí asegurada en la medida en que un Gobierno pueda simultáneamente mantener la legitimidad y promover el desarrollo socioeconómico. En este sentido, al plantearse las dimensiones subyacentes a la problemática de la gobernabilidad en América Latina, se ha señalado que éstas no son otras que "el fortalecimiento de la legitimidad del sistema político y de las instituciones estatales; y el desarrollo de la eficacia de las políticas públicas diseñadas e instrumentadas por el Estado" (Mayorga, 1992: 41). Y, en términos de Flisfisch: "para la consecución de la gobernabilidad no basta con la pura operación de las reglas del juego democrático... exige como resultado de la operación de esas reglas un orden" (Flisfisch, 1989: 117,118). Ese orden no es sino una situación de equilibrio social, generalizado y relativamente estable.

\section{BIBLIOGRAFIA CITADA}

Arbós, Xavier y Giner, Salvador (1993). La gobernabilidad. Ciudadanía y democracia en la encrucijada mundial . Siglo XXI. Madrid.

Birch, Anthony H. (1984). "Overload, Ungovernability and Delegitimation: the Theories and the British Case". British Journal of Political Science . 14. 135,160.

Bourricaud, Francois (1992). "Governance at the center and at the periphery". El Albala-Bertrand, Luis (coord.) (1992). Democratic culture and Governance. UNESCO/ Hispamerica. Gaithersburg. 35,49.

Buchanan, James M. y Wagner, Richard E. (1983). Déficit del sector público y democracia. Rialp. Madrid. Traducción de (1977). Democracy in Deficit: the political legacy of Lord Keynes. Academic Press. New York.

Coppedge, Michael (1993). "Institutions and Democratic Governance in Latin America". Ponencia preparada para la conferencia Rethinking Development Theories in Latin America. Institute of Latin American Studies. Uni-

(3) A pesar de que, precisamente, bajo ese marco la gobernabilidad es donde encuentra mayor conflicto, pues como señala Coppedge (1993: 11), "la gobernación y la democracia se basan en principios antagónicos estando abocados inevitablemente al conflicto. La gobernación requiere la efectiva representación de los grupos proporcionalmente a su poder: la demcoracia requiere la representación de los grupos en proporción al número de individuos que les apoyen. La gobernación respeta la lógica del poder mientras que la democracia respeta la lógica de la igualdad política". 
versity of North Carolina. Chapel Hill. 11,13 de marzo de 1993.

Crozier, Michel J., Huntington, Samuel J., y Watanuki, Joji (1975) The Crisis of Democracy. New York. New York University Press.

Flisfisch, Angel (1989). "Gobernabilidad y consolidación democrática: sugerencias para la discusión". Revista Mexicana de Sociología . LI.3 113,133.

Habermas, Jurgen (1978). Raison et legitimité . Payot. París.

Hirschman, Albert O. (1991). Retóricos de la intransigencia . Fondo de Cultura Económica. México.

Huntington, Samuel P. (1991). The Third Wave. Democratization in the Late Twentieth Century. University of Oklahoma Press. Norman.

Kohli, Atul (1990). Democracy and Discontent. India's Growing Crisis of Governability . Cambridge University Press. Cambridge.

Leca, Jean (1985). "Sur la gouvernabilité". En Leca y Papini (1985) (eds.) Les démocraties. Sont-elles gouvernables? Economica. Paris. 15,27.

Mayorga, René Antonio (Coord.) (1992). Democracia y Gobernabilidad en América Latina . Nueva Sociedad. Caracas. Nohlen, Dieter (1992). Sistemas electorales y gobernabili- dad. Working Paper 63. Institut de Ciéncies Poltiques i Socials. Barcelona.

O'Connor, James (1973). The fiscal crisis of the State. St.Martin's Press. New York.

Pasquino, Gianfranco (1988). "Gobernabilidad". En Bobbio, Mateucci y Pasquino. Diccionario de Política. Suplemento . Madrid. Siglo XXI.

Peters, B. Guy (1987). "Governability". En Bogdanor (1987). The Blackwell Encyclopaedia of Political Institutions. Basil Blackwell. New York. 256,257.

Schmitter, Philippe C. (1988). "La mediación entre los intereses y la gobernabilidad de los regímenes en Europa Occidental y Estados Unidos en la actualidad". En Berger, Suzanne (comp.) (1988). La organización de los grupos de interés en Europa Occidental . Ministerio de Trabajo y Seguridad Social. Madrid. 353-402.

Tomassini, Luciano (1993). Estado, Gobernabilidad y Desarrollo. Banco Interamericano de Desarrollo. Serie de Monografías 9. Washington.

\section{RESUMEN}

En este artículo, el autor se ocupa de conceptualizar lo que se entiende por gobernabilidad en el ámbito del sistema político.En primer lugar, se identifican minuciosamente los factores explicativos de la gobernabilidad para los diferentes análisis de las Ciencias Sociales. Estos son agrupados en cuatro apartados, de acuerdo con su carácter de variables independientes: 1) legitimidad y eficacia del sistema político en regímenes democráticos; 2) situaciones de crisis de los sistemas políticos; 3 ) el problema del déficit fiscal en los países occidentales industrializados, entendido desde distintas corrientes intelectuales; 4) los problemas de gobernación propios de los países en vías de desarrollo. En segundo y último lugar, se concluye que el tema de la gobernabilidad queda enmarcado exclusivamente en el ámbito gubernamental, y que su logro depende fundamentalmente de la capacidad efectiva del gobierno para mantener la legitimidad y promover el desarrollo socioeconómico, de un modo simultáneo.

\section{ABSTRACT}

In this article, the author is occupied in conceptualizing what is intended to be governability in the scope of the political system.First, explanatory factors of governability according to the different analysis of Social Sciences, are thoroughly identified.These indicators are organized into four groups, given their character of independent variables: 1)legitimacy and efficacy of the political system under democratic regimes; 2)situations of crisis of the political systems; 3 )the problem of the fiscal deficit in western industrialized countries, from the point of view of different intelectual currents; 4)the problems of governance propers to developing countries.In second and final place, the author concludes that the theme of governability stays limited exclusively to the governmental scope, and that its achievement depends fundamentally on the effective capacity of government for maintaining legitimacy and promoting socioeconomic development, in a simultaneous way. 